\section{Molecular \\ Syndromology}

Mol Syndromol 2014;5:87-99

DOI: $10.1159 / 000358504$

Published online: February 3, 2014

IT'S TIME TO

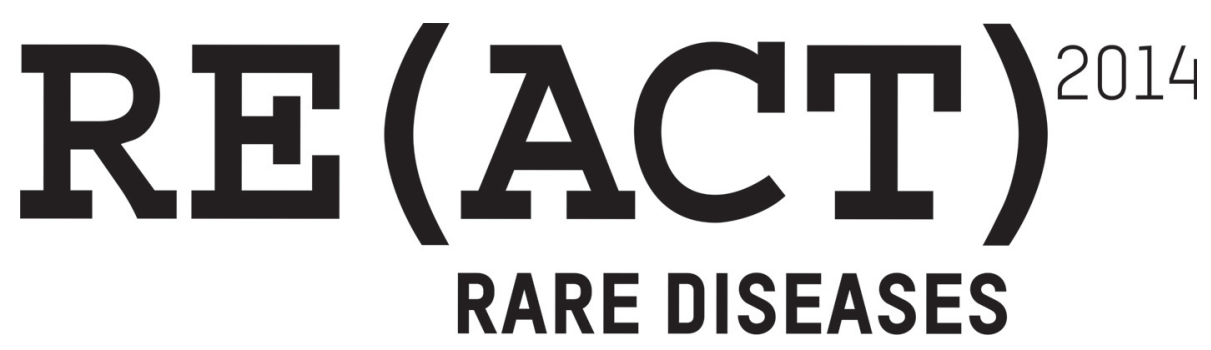

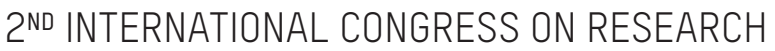

OF RARE AND ORPHAN DISEASES

$5^{\text {TH }}$ TO $8^{\text {TH }}$ MARCH 2014

GEHRY BUILDING, NOVARTIS CAMPUS, BASEL

INITIATED BY

\title{
BLACKSWAN FOUNDATION
}

Swiss Foundation for Research on Rare and Orphan Diseases

- G E B ERT R Ü F STIFT U N G WISSENSCHAFT.BEWEGEN

SUPPORTED BY
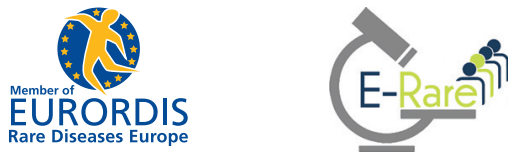

MAIN SPONSOR

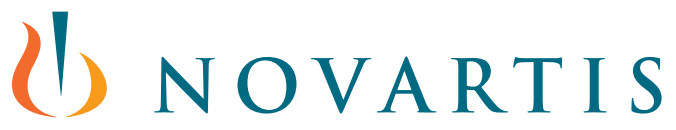

KR GR Basel · Freiburg $\cdot$ Paris $\cdot$ London $\cdot$ New York $\cdot$ Chennai $\cdot$ New Delhi . Bangkok $\cdot$ Beijing $\cdot$ Shanghai $\cdot$ Tokyo $\cdot$ Kuala Lumpur $\cdot$ Singapore $\cdot$ Sydney 


\section{Molecular Syndromology}

\section{Introduction}

Theyear 2014 is an important year for the BLACKSWAN Foundation (http://www.blackswanfoundation.ch) which aims to support the research on orphan and rare diseases. The RE(ACT) initiative, which is a 'knowledge-sharing' campaign, started in 2012 in Basel, Switzerland, with the first international congress focusing on research in the field of rare and orphan diseases (The RE(ACT) Congress 2012; http://www.react-congress.org). The first RE(ACT) Congress, organized in collaboration with the Gebert Rüf Stiftung (http://www.grstiftung.ch/en.html), brought together almost 300 people to discuss research into rare diseases (RDs) and the development of medicinal products to treat them. What made the congress unique was the interdisciplinary collaboration. Scientists from different disciplines - stem cell researchers, geneticists, biochemists, clinicians, and pharmacists - exchanged information with patient organizations.

A disease is considered rare in Europe by its incidence when it affects less than 1 in 2,000 citizens. Despite the rarity of each $\mathrm{RD}$ taken on its own, it is surprising to discover that altogether about 30 million people in the $27 \mathrm{EU}$ countries have a RD (http://whqlibdoc.who.int/hq/2004/ WHO_EDM_PAR_2004.7.pdf), meaning that $6-8 \%$ of the EU citizens are RD patients. This figure is equivalent to the combined populations of the Netherlands, Belgium and Luxembourg (http://www.eurordis.org/IMG/pdf/ princeps_document-EN.pdf, p 3).

RDs are also chronic and potentially fatal. Today, it is estimated that there are approximately 7,000 RDs; most of them have a genetic origin, and, in fewer cases, they are autoimmune and infectious.

RDs can affect anyone and especially children, as 3 out of 4 diseases present paediatric conditions and are often associated with severe disabilities and premature death.
The remaining fraction presents in adulthood, between 30 and 50 years of age. RDs can encompass a large variety of disorders and may affect one's ability to move (myopathies), to comprehend the world around us (fragile X syndrome), to breathe (cystic fibrosis), or to combat infection (immune deficiencies).

Because of the low individual prevalence, these diseases do not represent a public health priority for governments, and little research is performed. The market is so narrow for each disease that the pharmaceutical industry is reticent to invest in research and to develop new treatments.

There is a striking need for increased international cooperation in scientific research on RDs. Existing research efforts are in fact still scattered, and fragmented research is being performed with little coordination between research laboratories. This lack of coordination is particularly detrimental to the increase of knowledge on RDs because the resources are very limited and the patient population is small.

Medical and scientific knowledge about RDs is lacking. While the number of scientific publications about RDs continues to increase, particularly of those identifying new syndromes, less than 1,000 diseases benefit from a minimum of scientific knowledge, and these are essentially the 'most frequent' amongst RDs. The acquisition and diffusion of scientific knowledge is the vital basis for identification of diseases, and, most importantly, for research into new diagnostic and therapeutic procedures. In this context, exchange of experience is as important as expertise knowledge (http://www.eurordis.org/IMG/ pdf/princeps_document-EN.pdf, p 6).

Traditional funding mechanisms based on natural market conditions and access to public funds are not

\section{KARGER}

E-Mail karger@karger.com

www.karger.com/msy (c) 2014 S. Karger AG, Basel

$1661-8769 / 14 / 0052-0087 \$ 39.50 / 0$
Olivier Menzel

President and Founder of the BLACKSWAN Foundation

Via Cantonale 26, CH-6948 Porza (Switzerland)

http://www.blackswanfoundation.ch

E-Mail olivier.menzel@blackswanfoundation.ch 
adapted to the reality of research requirements on RDs. Specific public and private funding strategies and action plans for research on RDs are now an imperative.

BLACKSWAN Foundation in partnership with the Gebert Rüf Stiftung want to invert this with the RE(ACT) initiative. The main objective of the RE(ACT) initiative is to facilitate and accelerate the delivery of new pharmaceuticals and therapies for millions of patients affected by rare and orphan diseases and to create innovation in this sector. These goals will be pursued by the RE(ACT) Congress and the online RE(ACT) Community (http://www. react-community.org).

The RE(ACT) Congress is organized every 2 years and brings together leading researchers as well as young scientists working in different fields - from stem cell, cell biology, gene therapy, human genetic, or therapeutic ap- plications to present state-of-the-art research - to discuss results and to exchange ideas.

The RE(ACT) Community facilitates continuous collaboration between researchers on projects, as well as communication amongst patients and between patients and researchers, information gathering, identification of new funding mechanisms for research, and opportunities to optimize synergies between stakeholders from patient organizations to academic institutions, centres of expertise, health industry, regulators, and policy makers.

Through the RE(ACT) initiative, the two foundations encourage the creation of a society of researchers which can raise awareness about the needs of this field and emphasise the results achieved to gain stronger political leverage and ask for more support from public institutions. 


\section{Molecular Syndromology}

\section{A001 \\ Genetic Analysis of CAPS and TRAPS in Russian Patients Affected with Systemic Juvenile Idiopathic Arthritis}

\author{
L. Namazova-Baranova, K. Savostyanov, E. Alexeeva, A. Pushkov, \\ T. Sleptsova, D. Chistiakov, E. Suleymanov, A. Baranov \\ Molecular Genetic Diagnostics Department, Scientific Centre \\ of Children Health, Moscow, Russia
}

Background: Systemic juvenile idiopathic arthritis (JIA) is a rare subtype of JIA that can be classified as an autoinflammatory disease instead of an autoimmune disease. Methods: The study included 52 children ( 23 boys; 29 girls) at the age of 6 months to 17 years (mean $8.2(4.7 ; 11.5)$ ) with fever of unknown cause, after exclusion of infections, malignancy and autoimmune diseases. The median age at which symptoms began was $3.0(1.5 ; 5.1)$ years, mean disease duration was $4.4(1.0 ; 7.6)$ years. The commonest features were fever (100\%), arthritis or arthralgia (100\%), rash (96\%), hepato- and splenomegaly (96\%), lymphadenopathy (94\%), headache $(62 \%)$, abdominal pain $(58 \%)$, and eye manifestations (21\%). The patients were selected according to the clinical manifestations, with subsequent obligatory genetic counseling in the Division of Rheumatology of the Research Center for Children's Health. Patients' DNA was sequenced in all coding exons and intronic flanks of the TNFRSF1A and NLRP3 genes whose mutations cause autoinflammatory syndromes, TRAPS and CAPS, respectively. Results: In 7 patients, we found etiological mutations in TNFRSF1A. Most patients (6) had a mutation c.362G>A (p.R92Q) located in exon 4 and associated with the mild progression of TRAPS. Interestingly, 1 patient was homozygous for $\mathrm{p}$. R92Q while others were heterozygous. The seventh TRAPS patient had a novel frameshift mutation c.792delT (p.Lys265Serfs*87) in exon 9 of TNFRSF1A. In 2 patients, mutations in NLRP3 were detected. The first CAPS patient had a NLRP3 mutation c.2113C >A (p.Gln705Lys) whereas the second contained a novel mutation c.2861C $>\mathrm{T}$ (p.Thr954Met). Conclusions: Our analysis of the obtained data revealed a $17.3 \%$ incidence of CAPS and TRAPS in Russian systemic JIA patients.

E-Mail: docseasur@gmail.com

\section{A002 \\ Hunting for New Developmental Mutations with Dogs \\ M. Hytönen ${ }^{\text {a }}$ A. Lappalainen ${ }^{\mathrm{b}}$, M. Arumilli ${ }^{\mathrm{a}}$, P.-L. Lukinmaa ${ }^{\mathrm{c}}$, E. Sarkiala-Kesseld ${ }^{\text {, P. Nieminen }}{ }^{\text {d }}$, H. Lohi ${ }^{\text {a }}$ \\ aMolecular Neurology, Research Programs Unit and Department of Veterinary Biosciences, University of Helsinki and Folkhälsan Institute of Genetics, ${ }^{b}$ Equine and Small Animal Medicine, Faculty of Veterinary Medicine, 'Institute of Dentistry, and dVeterinary Teaching Hospital, University of Helsinki, Helsinki, Finland}

Background: Dogs have emerged as clinically and genetically relevant large animal models for human inherited disorders. Selective breeding has created more than 400 different breeds each representing genetic isolates with breed-specific morphological and behavioral characteristics as well as disease profile. The majority of the over 600 genetic disorders that have been described in dogs are similar to human conditions. Most of the canine disorders are Mendelian. Unique breed structure facilitates gene discovery and provides new candidate genes for corresponding human conditions. Methods: We have used various clinical and pathological techniques such as radiography and histology to characterize the novel canine phenotypes. To identify the genetic causes, we have applied both genome-wide association and whole-genome resequencing approaches. Results: We have established a large dog DNA bank with over 50,000 samples from 300 breeds. This provides a major resource for various genetic studies, including developmental disorders. We have initiated a significant multidisciplinary program to clinically and genetically characterize many congenital traits, including hip dysplasia, vertebral anomalies, craniomandibular osteopathy, mucopolysaccharidosis, chondrodysplasia, and dental anomalies. Here, we study 2 recessive, previously unknown disorders: a congenital syndrome characterized by severe mandibular prognathism, patellar subluxation and mental disturbances, and a disease with vigorous tooth attrition caused by abnormal hard tissue structure. Conclusions: The identification of the mutations causing these diseases will provide new candidate genes for corresponding human conditions, will establish the affected breeds as models to further explore disease mechanisms and develop therapeutic approaches. Furthermore, it will enable the development of genetic tests for breeding purposes in dogs. The 
presence of a large range of different breed-specific developmental defects in dogs provides a remarkable resource to facilitate gene discovery in rare disorders of comparative interest in human.

E-Mail: marjo.hytonen@helsinki.fi

\begin{tabular}{|c|}
\hline 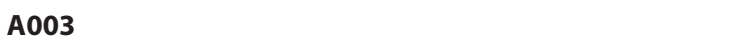 \\
\hline $\begin{array}{l}\text { Oorham's Disease of the Calvarium - } \\
\text { Complex Single-Step Skull Reconstruction }\end{array}$ \\
\hline V. Ohla ${ }^{\mathrm{a}, \mathrm{b}}$, A. Bayoumi ${ }^{\mathrm{a}}$, M. Hefty ${ }^{\mathrm{a}}$, M. Anderson ${ }^{\mathrm{a}}$, E. Kasper ${ }^{\mathrm{a}}$ \\
\hline $\begin{array}{l}\text { Division of Neurosurgery, Beth Israel Deaconess Medi } \\
\text { enter, Harvard Medical School, Boston, Mass., USA; } \\
\text { Department of Neurosurgery, University of Freiburg, } \\
\text { reiburg, Gerrmany }\end{array}$ \\
\hline
\end{tabular}

Background: Gorham's disease is a very rare osteolytic disorder characterized by uncontrolled proliferation of non-neoplastic vascular or lymphatic tissue, leading to progressive resorption and replacement of osseous matrix. Due to the low incidence, unknown cause and unpredictable natural history of the disease, a standardized treatment regimen has not yet been defined. Current treatments are experimental only as no single treatment has proven to be superior in arresting the course of the disease. Trials have included surgery, radiation and medical therapies employing drugs such as calcium salts, vitamin D supplements and hormones. A single-step surgery consisting of craniectomy for the diseased bone and reconstructive cranioplasty has not been described in the management of Gorham's disease. We therefore report our experience of a case with only substantial cranial involvement, which was successfully treated with excision of the diseased bone segment and a CAD/CAM custom-made cranioplasty implant to allow for synchronous repair. Clinical Presentation: A 25-year-old man with an asymptomatic skull defect over the left frontal convexity had noticed a progressive enlargement of the affected site. Physical examination revealed a clearly palpable depression of the frontotemporal bone. Conventional XR of the skull showed widespread loss of the native calvarial structure, corresponding to an osteolytic process. Subsequent MRI and CT scans disclosed the typical features of a patchy resorptive osteolysis concerning for an active underlying process. Due to the need for a histopathological diagnosis and the existing risk from a large unprotected area of his brain, surgical intervention was chosen. The goal was to obtain a pathological diagnosis as well as complex reconstruction of the afflicted area. A density graded CT scan was used to indicate the relative severity of the affected bone areas, and this information was used to design a custom-made cranioplasty allograft implant to allow for a single-step excisional craniectomy with synchronous repair. Pathological results revealed Gorham's disease and the patient has been recurrence-free for 2 years since surgery. Conclusion: Based on this report and available information in the literature, a single-stage surgery with excisional craniectomy and a custom-made cranioplasty allograft implant appears to be an effective therapy for patients suffering from Gorham's disease limited to vault involvement.

E-Mail: victoria.ohla@uniklinik-freiburg.de

\section{A004 \\ Is the Genetic Basis of Li-Fraumeni Syndrome Fully Deciphered?}

M. Akouchekian ${ }^{\text {a }}$, S. Hemati ${ }^{\text {b }}$

aDepartment of Medical Genetics \& Molecular Biology, Iran University of Medical Science, Teheran, and bepartment of Oncology, Isfahan University of Medical Science, Isfahan, Iran

Background: The definitions of predisposition syndromes for cancer have been characterized due to the occurrence of a nonrandom aggregation of cancers in families. Careful epidemiological studies are needed to distinguish between environmental and genetic causes, and in many cases this has confirmed the existence of inherited predisposition. Li-Fraumeni syndrome (LFS1; MIM 151623 ) is one of the cancer predisposition syndromes, which was initially proposed in 1969 and subsequently confirmed by a number of epidemiological studies. LFS is one of the most serious hereditary cancer syndromes with high risk of malignancy already in childhood. This syndrome is known as an autosomal dominant cancer predisposing syndrome due to a germline mutation in the p53 tumor suppressor gene. Methods: We describe a representative case of Li-Fraumeni syndrome. The patient is a 43 -year-old male who had osteosarcoma of mandible and a positive family history of cancer: his mother died at the age of 29 of brain cancer; his sister died at the age of 18 of breast cancer; his brother died at the age of 36 of liver cancer; and another sister died of leukemia at the age of 16. Complete sequence analysis of the TP53 gene in this patient was performed. After detailed analysis of the family history and medical records of affected individuals, we used standard diagnostic tools, e.g. sequencing and multiplex ligation-dependent probe amplification (MLPA), to analyze the TP53 gene in this family. Results: We performed whole gene sequencing for the TP53 gene. We detected a germline mutation in this patient that previously was reported as somatic mutation in LFS in the IARC database. Conclusions: Genomic modifications for tumor risk and genotype-phenotype correlations in LFS are still to be identified. We believe every new finding in this area can provide new insights into the pathogenesis and progression of Li-Fraumeni syndrome.

E-Mail: m-akouchekian@tums.ac.ir

\section{A005 \\ Enzyme Replacement Therapy for Homocystinuria \\ J.P. Kraus, E.M. Bublil, I. Park, T. Majtan, A.L. Salzman \\ Department of Pediatrics, University of Colorado School of Medicine, Aurora, Colo., USA}

Background: Cystathionine $\beta$-synthase (CBS)-deficient homocystinuria is the most common inherited defect of sulfur amino acid metabolism. CBS condenses homocysteine and serine to cystathionine, which is then cleaved to cysteine by cystathionine $\gamma$-lyase. Homocystinuria is characterized by very high levels of plasma Hcy, S-adenosylhomocysteine and reduced concentrations of cystathionine and cysteine. These abnormalities are manifested in thrombotic vascular disease, connective tissue aberra- 
tions and mental retardation. About $40 \%$ of the patients respond to pyridoxine therapy and the remaining ones are subjected to a low methionine diet supplemented with betaine to promote the re-methylation of homocysteine to methionine. This therapy is difficult to comply with and has little impact on downstream metabolites. We envisioned that administration of recombinant CBS to the circulation may positively impact the cellular equilibrium of sulfur amino acids. Methods: We have expressed and purified human truncated CBS in E. coli and PEGylated it. The enzyme was injected subcutaneously to our HO mouse model of homocystinuria at a dose of $5 \mathrm{mg} / \mathrm{kg}$ weight. Results: We found that administration of a PEGylated truncated human CBS enzyme to homocystinuric mice resulted in a 80\% decrease in Hcy, 900\% increase in cystathionine, and normalization of cysteine concentrations, indicative of re-activation of the intracellular transsulfuration pathway. Conclusions: The data strongly suggest that CBS enzyme replacement therapy is a promising approach for treatment of homocystinuria, and that ERT for metabolic deficiencies may not necessitate introduction of an enzyme into its native intracellular environment.

E-Mail: jan.kraus@ucdenver.edu

\section{A006 \\ Rad50 Phosphorylation Promotes ATR Downstream Signaling and DNA Restart Following Replication Stress}

M. Gatei ${ }^{\mathrm{a}}$, A.W. Kijas ${ }^{\mathrm{a}}$, M.F. Lavin ${ }^{\mathrm{a}, \mathrm{b}}$

${ }^{a} Q$ IMR Berghofer Medical Research Institute, Radiation Biology and Oncology, Brisbane, and buniversity of Queensland Centre for Clinical Research, Herston, Australia

Background: To investigate the role of Rad50 in the cellular response to DNA damage following DNA replication fork stalling. Methods: Control (NFF), ATM-null (AT5), ATR-Seckeland Rad50-deficient (F239) h-TERT immortalized fibroblasts were exposed to either $5 \mathrm{~Gy}$ of IR, $20 \mathrm{~J} / \mathrm{m}^{2}$ of UV or $2 \mathrm{mM}$ of $\mathrm{HU}$ and harvested after $15 \mathrm{~min}, 1 \mathrm{~h}$ or $2 \mathrm{~h}$, respectively. These cells were employed for immunoblotting, ATR kinase assay, G2/M checkpoint activation, clonogenic survival and immunofluorescence analyses. Results: The Mre11/Rad50/NBN complex plays a key role in DNA damage signalling, cell cycle checkpoint control and DNA repair through ataxia telangiectasia mutated (ATM) and ataxia telangiectasia mutated and Rad3 related (ATR) activation in response to DNA damage. Hypomorphic mutations in these genes give rise to ataxia telangiectasia-related disorder (ATLD) Mre11-deficient, Nijmegen breakage syndrome (NBS) NBN-deficient and Nijmegen breakage syndrome-like disorder (NBSLD) due to Rad50 deficiency. These disorders are characterized by cellular radiosensitivity, cell cycle abnormalities and a defective response to DNA damage. Although several families with ATLD and NBS have been identified, only 2 patients with Rad50 deficiency have been reported. We provide evidence here that $\operatorname{Rad} 50$ is required for ATR activation and Rad50 is phosphorylated by ATR at $\mathrm{S} 635$ following replication stress. This activation is required to phosphorylate Chk1 at S317 and a Rad50 S635G mutant failed to do so. This indicates that Rad50 acts upstream as an activator as well as downstream as an effecter for ATR in response to stalled replication forks. Interestingly, we find that Rad50 phosphorylation is essential for DNA replication restart and the mechanism involved is by promoting loading of cohesin at sites of DNA replication restart. We also demonstrate that replication stress-induced Rad50 phosphorylation is functionally significant for cell survival and cell cycle checkpoint activation. These results highlight the importance of $\operatorname{Rad} 50$ in the aspects of the DNA response to replication stress. Conclusions: These results demonstrate that Rad50 mediates ATR-dependent substrate phosphorylation in response to replication stress. Furthermore, these data also indicate that Rad50 is phosphorylated at a single site (S635) to play the role in cell cycle checkpoint activation and DNA replication restart.

E-Mail: magtoufG@qimr.edu.au

\section{A007 \\ Copy Number Analyses in Five Monozygotic Discordant Twin Pairs Reveal MMP14, LRP10 and Genes Relevant for Extracellular Matrix and Neoangiogenesis as Candidate Genes in Uterovaginal Aplasia (MRKH Syndrome)}

K.K. Ralla , S. Eisenbeis ${ }^{\mathrm{a}}$, G. Barresi ${ }^{\mathrm{a}}$, M. Walter ${ }^{\mathrm{b}}$, S. Poths ${ }^{\mathrm{b}}$, D. Wallwiener ${ }^{\mathrm{a}}$, O. Riess ${ }^{\mathrm{b}}$, S. Brucker ${ }^{\mathrm{a}}$, M. Bonin ${ }^{\mathrm{b}}$

Departments of abstetrics and Gynecology, and ${ }^{b}$ Medical Genetics, Microarray Facility, University Hospital, Tübingen, Germany

Background: The Mayer-Rokitansky-Küster-Hauser syndrome (MRKHS) affects at least 1 in 4,500 female live births. It is characterized by vaginal and uterine aplasia in individuals with a normal 46,XX karyotype and normal secondary characteristics. The syndrome has been considered as sporadic anomaly for a long time, but familial clustering occurs and supports the hypothesis of a genetic origin. However, the identification of any locus using standard genetic linkage analysis was impossible so far, and cases of monozygotic, discordant twins challenged the purely genetic basis. Methods: In our study, we had the unique opportunity to genetically analyze 5 pairs of monozygotic twins discordant for MRKHS; comprising the advantage that already 1 pair of twins can reveal the genetic cause of the syndrome. We used copy number variations (CNVs) in order to identify differences between the affected and unaffected twin. Results: In total, $64 \mathrm{CNVs}$ were detected, but none of the CNVs was differential between the discordant twin pairs. Additional detailed analysis revealed differences in CNV-patterns in 1 out of the 5 twin pairs which have never been listed within the Database of Genomic Variants. In twin 1, who had MRKH type II with unilateral kidney agenesis and a duplicated ureter, we identified 3 duplications and 1 deletion. None of the deleted genes had any known relevance for the development of the female genital tract. However, the duplication found on chromosome 14 comprised 2 genes, namely MMP14 and LRP10, which have known functions within uterine endometrium and embryology. Additional network analysis revealed important connections to differentially expressed genes in $\mathrm{MRKH}$ patients found in earlier studies. These genes include several molecules involved in remodeling of the extracellular matrix like 
MMP14, SPOCK3, ITIH5, and PI3K, and neoangiogenesis like VEGFC, KDR, FLT1, and neuropilin. Conclusion: The occurrence of these clusters gives evidence of a deficiency in vascularization during uterine development and/or disturbed reorganization of ECM components especially during elongation of the Müllerian duct influenced by the embryologically relevant (PI3K)/AKT pathway in the network. This scenario allows us to reason that the genes are probably involved in the onset of the anomaly. Therefore these genes could be seen as new candidates for MRKHS.

E-Mail: katharina.rall@med.uni-tuebingen.de

\section{A008 \\ Epigenetic Differences as a Causing Mechanism of the MRKH Syndrome: Analysis of Differentially Expressed microRNAs in Uterine Rudiments of MRKH Patients Compared with Uterine Tissue from Controls} S. Eisenbeis ${ }^{\mathrm{a}}$, V. Henninger ${ }^{\mathrm{a}}$, D. Rückner ${ }^{\mathrm{a}}$, M. Walter ${ }^{\mathrm{b}}$, S. Poths ${ }^{\mathrm{b}}$,
D. Wallwiener ${ }^{\mathrm{a}}$, O. Riess ${ }^{\mathrm{b}}$, S. Brucker ${ }^{\mathrm{a}}$, M. Bonin ${ }^{\mathrm{b}}$, K.K. Rall $^{\mathrm{a}}$

Departments of abstetrics and Gynecology, and ${ }^{\mathrm{b}}$ Medical Genetics, Microarray Facility, University Hospital, Tübingen, Germany

Background: The Mayer-Rokitansky-Küster-Hauser syndrome (MRKHS) is a malformation of the female genital tract characterized by congenital aplasia of the uterus and the upper part of the vagina. It is present in at least 1 out of 4,500 women with functional ovaries and a normal 46,XX karyotype. To date the etiology is largely unknown. In a previous study, where we used a combined whole-genome expression and methylation approach, we came to the hypothesis that either deficient estrogen receptors or the ectopic expression of certain HOXA genes could lead to this malformation. Epigenetic causes are further supported by the existence of 5 monozygotic, phenotypically discordant twin pairs in our collective. Methods: In order to determine differences on the level of gene expression between MRKH patients and non-MRKH controls, microRNA-sequencing experiments were carried out. Uterine rudiments of $10 \mathrm{MRKH}$ patients and uterine tissue samples from 10 control patients were obtained and used for microRNA isolation. Differentially expressed microRNAs between both groups were detected by sequencing using Illumina Genome Analyzer IIx. The validation of these results was performed by qRTPCR. Results: The sequencing revealed 33 significantly differentially expressed microRNAs. Associated candidate genes coincide partly with genes identified in the previous whole-genome and methylation approach. All results were validated by qRT-PCR. After comparing the preliminary data and the current literature, 5 microRNAs are in a special focus, with 4 being significantly downregulated in MRKH patients and 1 significantly upregulated. These candidate genes such as ESR1, FGF7, SPOCK3, and HOXA5 are known to play an important role in embryonic kidney and uterine development and the onset of menarche. Conclusion: With the analysis of differentially expressed microRNAs, we were able to confirm some of the previously described candidate genes for MRKHS and to identify new genes likely involved in the onset of the syndrome. Currently pending trials, such as the UTR cloning, will provide further insights into their influence of the disease origin.

E-Mail: simone.eisenbeis@med.uni-tuebingen.de

\section{A009 \\ Further Molecular Characterization of a Novel Neurodegenerative Syndrome Associated with a Mutation in the Seipin/BSCL2 Gene}

\section{Araujo-Vilar, A. Ruiz-Riquelme, S. Sanchez-Iglesias, J.R. Requena}

CIMUS Biomedical Research Institute, Santiago de Compostela, Spain

Mutations in the seipin gene (BSCL2) cause either type 2 congenital generalized lipodystrophy (BSCL) or dominant motor neuron diseases. However, we recently discovered a c.985C $>\mathrm{T}$ mutation in the BSCL2 gene that results in a novel fatal neurodegenerative syndrome (Celia encephalopathy). This mutation induces an alternative splicing site which results in skipping of exon 7 and a reading frame shift. Homozygous patients suffer from progressive encephalopathy from the age of 2-3 years, with fatal outcome at age 6-8 years; however, carriers for the c.985C>T mutation are asymptomatic, conflicting with the gain of toxic function attributed to the mutation. Our previous studies showed a partial nuclear localization of wild type (wt) and exon 7-skipped seipin. This location might be related to the presence of intranuclear ubiquitin-positive inclusions in brain tissue from a homozygous patient (index case), which are likely to consist of misfolded, aggregated mutant seipin. Besides, ER stress in cells expressing exon 7-skipped seipin was also found. Here, we report further molecular characterization of exon 7-skipped seipin. Using density gradient ultracentrifugation, we found that wt seipin oligomerizes forming tetramers according to calibration with a set of proteins of known MW. We also found that exon 7-skipped seipin forms much larger aggregates under similar conditions, corresponding to dodecamers. We hypothesized that wt and exon 7-skipped seipin might interact and form mixed oligomers. Given that levels of wt seipin expressed by heterozygous individuals are substantially higher than those of exon 7-skipped seipin, we reasoned that wt seipin might rescue the phenotype by recruiting exon 7 -skipped seipin into mixed normal size oligomers, thus impeding their aggregation into larger, presumably toxic, oligomers. We therefore expressed wt and exon 7-skipped seipin at a 3:1 ratio in HeLa cells and performed density gradient ultracentrifugation of cell extracts. We found a change in the pattern of distribution of each of the seipin isoforms, with exon 7 -skipped seipin emerging at earlier fractions. This result clearly indicates the interaction between both seipin isoforms through the formation of mixed oligomers. Together, our findings provide a clue about the origin of the intranuclear aggregates observed in neurons from the index case, and offer a possible explanation for the absence of phenotype in heterozygous mutation carriers.

Funded by Consellería de Industria (Xunta de Galicia) 10PXIB208013PR and ISCIII-FEDER PI10/02873.

E-Mail: david.araujo@usc.es 


\section{A010}

\section{An Epidemiological Survey of Adults with Histiocytic} Disorders in the Northeast Region of the UK

\author{
J. Salotti ${ }^{\mathrm{a}}$, K. Wood ${ }^{\mathrm{d}}$, V. Bigley ${ }^{\mathrm{b}}$, M. Collin ${ }^{\mathrm{b}}$, K. Windebank ${ }^{\mathrm{c}}$ \\ Institutes of a Health \& Society, ${ }^{b}$ Cellular Medicine, and \\ 'Northern Institute for Cancer Research, Newcastle University,

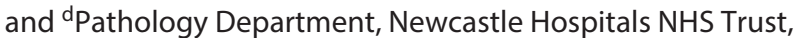 \\ Newcastle upon Tyne, UK
}

Background: Histiocytic disorders have clinical and pathological features of neoplasia and inflammation and may cause dysfunction of multiple organ systems. Adults may therefore present to many different clinical specialties. However, there is no national or international consensus on the best treatment and no open clinical trial or national registry. The scale of the problem in the UK is unknown. Some adults in the Northeast region attend a dedicated clinic in Newcastle with haematological, dermatological and paediatric ( $\mathrm{LCH}$ expert) input, but it is not known which other specialists are treating patients. This regional study will estimate the frequency of disease and assess the treatments and services patients are receiving. This may then lead to the establishment of a national registry for those with histiocytoses. Methods: Patients are being identified via pathology department databases by searching for appropriate Snomed codes (in brackets) for histiocytic diseases: Langerhans cell histiocytosis - LCH (M7791, M77800); haemophagocytic lymphohistiocytosis - HLH (F42000); Rosai-Dorfman - R-D (M77940); Erdheim Chester - ECD (M77800); juvenile xanthogranuloma -JX (M55380). Eligible patients are $\geq 16$ years old and diagnosed by biopsy from 2000-2012 with a histiocytic disease. For those eligible, questionnaires are mailed to the referring clinicians for information on patient presentation, treatment and services used. The study received appropriate research governance approvals. Results: A pilot study in one regional hospital (Newcastle) identified 67 adults, aged 16-81 years. However, 20 cases were found not to have a histiocytic disease and a further 10 were ineligible for other reasons. Of those with histiocytosis, 25 had LCH, 7 had JX, 4 had R-D disease and 1 had HLH. The M:F ratio was $1.5: 1$. Of those with $\mathrm{LCH}$, the sites of disease were lung, bone, lymph, skin and genital mucosa. A further 28 cases have been identified at 2 of 8 other regional hospitals, including 18 cases of JX. Conclusion: Snomed codes were found to include reactive types of histiocytosis as well as primary histiocytic disease. Therefore, anonymised pathology reports are now being screened to exclude ineligible patients before contacting clinicians. Data collection is continuing at the other regional hospitals. As well as improving our knowledge, it is hoped that the results of the study will improve co-operation and planning for adults with histiocytoses both regionally and nationally.

E-Mail: jane.salotti@ncl.ac.uk

\section{A011 \\ Diagnosis of Familial Dysautonomia in Europe - The Need for Increased Awareness}

C. Maayan ${ }^{\text {a }}$ N. Holzer ${ }^{\mathrm{a}}$, G. Belitsky ${ }^{\mathrm{d}}$, K. Jacobs ${ }^{\mathrm{a}}$, O. Levzion-Korach', S. Khoury ${ }^{\text {b }}$

Departments of apediatrics, ${ }^{\text {b Neurology, }}$ CHospital

Management, and dSocial Work, Israeli Familial Dysautonomia

Center, Center for Autonomic Diseases, Hadassah Medical

Center Mount Scopus, Jerusalem, Israel

Background: Familial dysautonomia (FD) is a severe, complicated autosomal recessive disorder affecting Ashkenazi Jews. The carrier frequency of the FD mutation in Ashkenazi Jews is 1:17 to $1: 32$ with a birth incidence of $1: 3,703$. The diagnosis of FD requires awareness of typical clinical features, e.g. feeding difficulties, vomiting, aspiration pneumonia, blood pressure instability, hypotonia, absence of overflow tears, kyphoscoliosis and more. Since 2001, FD diagnosis has also been made possible by genetic testing. As FD is a rare disease, we predict that there could be many patients who are not diagnosed, and we therefore estimated the numbers in Europe. Methods: According to the FD frequencies, we calculated the estimated FD patients who were expected to be diagnosed according to estimated Ashkenazi Jews in European countries, based on the information from the website http://Jewishvirtuallibrary. org/jsource/Judaism/jewpop.html. As it is not possible in a few countries to distinguish between Ashkenazi and Sephardic Jews, we have eliminated Spain, Portugal and France where Sephardic Jews are the majority. Mixed marriages also affect the statistics. Results: Based on the number of FD birth incidence, more affected FD patients should have been born. It is estimated that in Europe during 2012 there were 919,800 Ashkenazi Jews. However, there are only 31 diagnosed FD patients out of a calculated 248.3 FD patients (12.5\%). Four patients who were born in Russia were diagnosed in Israel, where there is greater awareness of FD. Conclusion: The 4 undiagnosed FD cases that migrated to Israel and the small number of documented FD cases in Europe indicate fewer FD cases than estimated and suggest that FD may be misdiagnosed, unreported or both. An unknown number of affected fetuses may have been terminated. Assimilation of Jews might also decrease the number; however, mixed origin can also result in birth of FD patients as both parents can be carriers. Increased FD awareness and genetic screening is needed.

E-Mail: cmaayan@hadassah.org.il

\section{A012 \\ Elimination of HIV Sequences from Lentiviral Gene Therapy Vector DNA Delivered to Target Cells: The LTR-1 System}

\author{
S.J. Howe, C.A. Vink \\ UCL Institute of Child Health, London, UK
}

Aim: To reduce the amount of remaining HIV-1 sequence from lentiviral gene delivery vectors to improve safety and efficacy of the viruses for gene therapy of rare diseases. Background: Lentiviral vectors (LV) are useful tools for gene therapy approaches to treat- 
ing rare diseases. Current vectors based on HIV-1 effectively deliver genes to a range of cells, but current clinically approved viral backbones contain almost $20 \%$ wild-type HIV sequence. LV packaging sequences ( $\Psi$, Rev Response Element) up to $2 \mathrm{~kb}$ long are necessary for viral RNA genome packaging into viral particles in producer cells. In standard LVs, these sequences are reverse transcribed into DNA and persist in target cells after transduction. This persistence creates several known and potential problems for LV gene therapy applications. Splice sites within packaging sequences have been shown to splice with nearby host genes, creating aberrant fusion transcripts. The $\mathrm{CpG}$ island within the packaging sequence undergoes DNA methylation in some target cells, potentially contributing to transgene silencing. Packaging sequences enable remobilisation of $\mathrm{LV}$ genomes in cells expressing viral proteins, which could be problematic in HIV-positive patients. Large packaging sequences within the reverse transcript may reduce the size of the transgene cassette which can be accommodated. Results: In standard LVs, packaging sequences are located between the 2 viral long terminal repeats (LTRs), within the region of the vector that is reverse transcribed. We have developed a novel 'LTR-1' transfer vector in which the packaging sequences are located downstream of a single $3^{\prime}$ LTR, so are present in the RNA genome during virion packaging but are outside of the region of the genome that is reverse transcribed into DNA in the target cell. For the first time these minimal HIV sequence vectors can now be produced at high titre $\left(3 \times 10^{8} \mathrm{TU} / \mathrm{ml}\right.$ by eGFP flow cytometry of $293 \mathrm{Ts}$, pCCL parallel preparation $1 \times 10^{9} \mathrm{TU} / \mathrm{ml}$ ) and the proportion of eGFP+ cells is stable between 3 and 14 days post-transduction. Conclusion: This configuration eliminates most of the remaining viral DNA delivered into target cells and could be a feature of the next generation of gene therapy vectors based on HIV-1 and other retroviruses.

E-Mail: s.howe@ucl.ac.uk

\section{A013 \\ Tissue Engineering Approaches and Non-Invasive Optical Tools to Address the Diagnosis and Therapy of Rare Diseases in Women}

\author{
E. Brauchle ${ }^{\mathrm{a}, \mathrm{b}}$, S. Hinderer ${ }^{\mathrm{b}}$, M. Votteler ${ }^{\mathrm{a}}$, S. Eisenbeis ${ }^{\mathrm{a}}$, K.K. Rall $^{\mathrm{a}}$, \\ D. Wallwiener ${ }^{\mathrm{a}}$ S. Brucker ${ }^{\mathrm{a}}$, K. Schenke-Layland ${ }^{\mathrm{a}-\mathrm{c}}$ \\ aUniversity Woman's Hospital Tübingen, Eberhard Karls \\ University, Tübingen, and 'bepartment of Cell and Tissue \\ Engineering, Fraunhofer Institute for Interfacial Engineering \\ and Biotechnology (IGB), Stuttgart, Germany; ' Department of \\ Medicine/Cardiology, Cardiovascular Research Laboratories, \\ David Geffen School of Medicine at UCLA, Los Angeles, Calif., \\ USA
}

Female rare diseases are often connected to individual forms of genital malformations such as Mayer-Rokitansky-Küster-Hauser (MRKH) syndrome, which is the congenital absence of the vagina and uterus, occurring in approximately 1 of 4,500 female live births. While several projects are ongoing worldwide, the etiology for most female genital malformation is still unknown. The Women's University Hospital in Tübingen successfully established surgical therapies to correct certain malformations, but in complex malformations like cervical and partial vaginal aplasia, there is still no ideal implant that could replace missing or malformed genital tissues in women. Our goal is to design implantable tissue equivalents that can support or replace malformed female sex organs. We aim to illuminate pathways that are responsible for uterovaginal agenesis and other malformations and which can be translated into tissue constructs with stem cell technologies. We will characterize healthy vaginal and uterine tissue, analyzing their biomechanical and functional properties, which are determined by the interaction of extracellular matrix (ECM) and specific cell phenotypes. Key proteins of the ECM will be identified and will serve as constituents to manufacture new biomaterials by electrospinning or bioprinting technologies. These processes enable the design of biomaterials with complex multi-layered compositions, defined fiber alignments and pore sizes; parameters that are related to biocompatibility and cell-material interactions of the manufactured materials. In addition, we will investigate the use of non-invasive optical technologies that can assess biochemical information of healthy and in vitro-constructed genital tissues. Multiphoton imaging visualizes the 3-dimensional organization of collagen as well as elastin fibers and can characterize cells based on their intrinsic fluorescent metabolites. Raman spectroscopy, another laser-based technique, reflects the global molecular constitutions within tissues and can identify ECM components. Due to their non-invasive character, both Raman spectroscopy and multiphoton imaging could potentially serve as diagnostic tools to detect tissue remodeling processes, which are related to rare disease in women. Ultimately, our findings will assist in the development of new diagnostic tools and therapies for women affected with uterovaginal agenesis and other genital malformations and help to identify the underlying etiology.

E-Mail: eva.brauchle@igb.fraunhofer.de

\section{A014 \\ Molecular Characterization of the Only Frequently Recurring Mutation in Carbamoyl Phosphate Synthetase 1 (CPS1) Deficiency}

L. Hu ${ }^{\mathrm{a}, \mathrm{b}}$, C. Diez-Fernandez ${ }^{\mathrm{c}, \mathrm{d}}$, V. Rüfenachta ${ }^{\mathrm{a}, \mathrm{b}}$, V. Rubio $^{\mathrm{c}, \mathrm{d}}$, J. Häberle ${ }^{a, b}$

aDivision of Metabolism, University Children's Hospital,

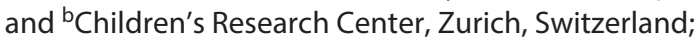
'Instituto de Biomedicina de Valencia of the CSIC, and

dGroup 739, Centro de Investigación Biomédica en Red para

Enfermedades Raras (CIBERER-ISCIII), Valencia, Spain

Background: Carbamoyl phosphate synthetase 1 deficiency (CPS1D), due to CPS1 mutations, is a rare autosomal-recessive urea cycle disorder causing hyperammonemia that can lead to death or severe neurological impairment. CPS1 catalyzes carbamoyl phosphate formation from ammonia, bicarbonate and 2 ATPs, and requires the essential allosteric activator $\mathrm{N}$-acetyl-L-glutamate. Clinical mutations were found spreading over the entire CPS1 coding region, occurring mainly in single families, with little recurrence. We characterize here the only known frequently recurring CPS1 mutation, p.Val1013del, found in patients of Turkish descent. Materials and Methods: p.Val1013del was found in 8 unrelated Turkish CPS1D patients. Recombinant, His-tagged CPS1, 
either wild-type (WT) or V1013del, was expressed using baculovirus/insect cells (confirmed by western blotting of virus-infected cell extracts). These CPS1 forms were purified to homogeneity. The global CPS1 reaction and the ATPase and ATP synthesis partial reactions that reflect, respectively, the bicarbonate and the carbamate phosphorylation steps, were assayed. Structural modeling was performed to rationalize the p.Val1013del effects. Results: CPS1 WT and V1013del mutant were expressed at comparable level and were purified to homogeneity (Coomassie-stained SDSPAGE). The mutant exhibited no significant residual activities in the assays for the carbamoyl phosphate synthesis reaction or for the partial reactions. In the CPS1 structural model, V1013 belongs to a highly hydrophobic $\beta$-strand at the middle of the central $\beta$-sheet of the A subdomain of the carbamate phosphorylation domain (an ATP-grasp domain). It is close to the predicted carbamate tunnel that links both phosphorylation sites, being just 5 residues away from E1018, which is predicted to belong to the tunnel wall. Conclusion: p.V1013del inactivates the enzyme but does not render the enzyme grossly unstable or insoluble. The deletion, by shortening the $\beta$-strand, could pull E1018 away from the carbamate tunnel, distorting this tunnel and possibly hampering the connection between both phosphorylation steps.

E-Mail: Liyan.hu@kispi.uzh.ch

\section{A015 \\ Lamin B1 Modulates Cell Fate Commitment and Differentiation in Murine-Derived Neural Stem Cells}

\section{S. Mahajani, C. Giacomini, L. Gasparini}

Department of Neuroscience and Brain Technology, Italian Institute of Technology, Genova, Italy

Lamin B1 (LMNB1) is a type V intermediate filament protein and is one of the major components of the nuclear lamina. It has been shown that $L M N B 1$ acts as a modifier gene affecting the risk of neural tube defects in mice and humans, in which synonymous and non-synonymous missense variants of $L M N B 1$ resulting in protein loss-of-function have been observed. Consistently, it has been demonstrated that experimental ablation of LMNB1 deeply impairs neurogenesis during embryonic development in the mouse. However, how LMNB1 protein expression regulates neurogenesis at the cellular and molecular levels is still undetermined. Here, we investigate whether LMNB1 influences cell fate commitment and early neuronal differentiation in cultured neural stem cells (NSCs) from the dorsal forebrain of E12 embryos of wild type (WT) and LMNB1-null mice. We find that the NSC differentiation rate into neurons and astrocytes, but not oligodendrocytes, is dependent on LMNB1 protein levels. In fact, LMNB1 deficiency significantly reduces the number of neurons and increases the number of astrocytes originating from NSCs, without altering oligodendrocyte numbers. Conversely, LMNB1 overexpression significantly increases the rate of NSC differentiation into neurons, but does not change the numbers of both astrocytes and oligodendrocytes. Apoptotic features are undetectable in NSC-derived cells, indicating that the observed changes are not due to altered cellular survival. Nuclear abnormalities (i.e. reduced nuclear area and circularity) are present only in LMNB1-deficient NSC-derived neurons, but not astrocytes and oligodendrocytes, nor
LMNB1-overexpressing neurons. We also find that LMNB1 protein levels affect the differentiation of NSC-derived neurons. In fact, both LMNB1-deficiency and overexpression significantly alter axonal length of NSC-derived neurons, indicating that the protein levels of LMNB1 regulate axonal outgrowth. These results indicate that LMNB1 plays a key role in cell fate commitment and differentiation during embryonic neurogenesis and that finely tuned levels of the protein are required to achieve proper morphological properties of NSC-derived neurons.

This work is funded by a grant from the European Leukodystrophy Association to L.G.

E-Mail: sameehan.mahajani@iit.it

\section{A016 \\ Alterations of Lamin B1 Levels Affect Viability and Differentiation of Primary Murine Cortical Neurons}

C. Giacomini, S. Mahajani, A. Contestabile, R. Marotta, R. Ruffilli, L. Gasparini

Department of Neuroscience and Brain Technology and Nanobiotech Facility, Italian Institute of Technology, Genova, Italy

Lamin B1 (LMNB1) is one of the major components of the nuclear lamina, a filamentous network underlying the inner nuclear envelope of eukaryotic cells. There is evidence that LMNB1 is essential during embryonic development of the mouse brain and plays a key role in maintaining brain function in humans during adulthood. In fact, it has been shown that neurogenesis and cortical development are severely impaired in LMNB1-deficient mice. Moreover, in humans, duplications of the LMNB1 gene on chromosome 5 are associated with adult-onset autosomal dominant leukodystrophy (ADLD), a rare demyelinating disease with neurodegenerative features. This suggests that the brain is particularly vulnerable to altered levels of LMNB1. Here, we investigate how altered protein levels of LMNB1 affect neuronal viability and morphology in vitro. We found that in LMNB1-deficient primary cortical neurons, the nuclear area is reduced and nuclear pore complexes are delocalized. LMNB1 overexpression in neurons results in abnormal nuclei, with blebbings similar to those detected in primary human skin fibroblasts derived from ADLD patients. Abnormal LMNB1 levels also affect survival and differentiation of neurons. In fact, in cortical neurons, LMNB1 deficiency significantly increases the percentage of apoptotic nuclei. LMNB1 transient overexpression also induces apoptosis at early, but not late differentiation stages. LMNB1 protein levels also profoundly affect the morphology of surviving neurons. LMNB1-deficient neurons have reduced dendritic length and arborisation, while those overexpressing LMNB1 have reduced axonal outgrowth. Overall, our data indicate that altered LMNB1 expression levels deeply affect neuronal survival and morphology, with distinct outcomes at different timing of neuronal differentiation.

This work is funded by grant GGP 10184 from the Italian TELETHON Foundation to L.G.

E-Mail: caterina.giacomini@iit.it 


\section{A017 \\ Canine Models of Human Rare Diseases \\ H. Lohi}

Department of Molecular Neurology, Research Programs Unit, Helsinki, Finland

Background: The annotation of the canine genome and subsequent development of new genomic tools have established purebred dogs as a powerful model for gene discovery. Over 600 genetic conditions, often enriched in certain breeds, have been described and many of them are Mendelian and represent clinically relevant models to corresponding human disorders. We have established a large collaborative genomic program to establish resources and identify the genetic causes in various disease groups. We have collected blood samples in over 50,000 dogs in 300 breeds with more than 100 phenotypes in various disease groups, including neurological, vision, autoimmune, musculoskeletal, metabolic, and behavioral conditions. Our aim is to discover the clinical and genetic backgrounds of the canine conditions as models for the human disorders. Methods: Clinical diagnoses have been established through multiple protocols in collaboration with specialized veterinarians. We have established large pedigrees with the help of a public registry to define the modes of inheritance of the conditions, to establish proper study design for genetic analyses. To identify the genetic causes behind the disorders, we have used canine-specific high-density SNP arrays, exome or whole-genome resequencing methods. Various bioinformatics, statistical and validation methods have been used to map new disease loci and confirm candidate mutations. Results: We have identified mutations in canine syndromic and idiopathic epilepsies, progressive ataxia, progressive retinal atrophy, various storage diseases (Pompe disease, MPS VII), and chondrodysplasia. In addition, we have mapped new loci for late-onset glaucoma, different neurobehavioral traits such as noise and social phobia, skeletal conditions including hip dysplasia and dwarfism. Our ongoing exome and resequencing studies aim to establish a significant sequence and variant database to further facilitate gene discoveries in many conditions. Conclusions: Our results have several implications. First, we have identified new candidate genes and pathways for mutation screenings in human patients. Second, we have discovered new disease mechanisms and developed new genetic tests for breeding programs. Finally, gene discoveries have established large animal models for the development of novel therapies, such as our ongoing collaborative gene therapy effort in the Pompe disease.

E-Mail: hannes.lohi@helsinki.fi

\section{A018 \\ Partnerships for New Medicines - Repositioning for the Treatment of ALS}

K. Kaloulis, E. Vom Baur, D. Toronto, M. Dreano

ReMedys Foundation, Geneva, Switzerland

To overcome the gap between academia-originating, promising therapeutic opportunities without commercial interest on one side, and industrial partnering, clinical development and availability to patients on the other side, we founded the ReMedys Foundation. ReMedys is a not-for-profit, patient-centric entity, based in Geneva, Switzerland, committed in developing innovative therapeutic ideas into clinically testable therapies for patients with severe disease without effective treatment - independent of commercial potential. ReMedys has been founded by seasoned industry experts and maintains a broad network of pharmaceutical industry experts in all fields required for effective drug development. ReMedys is directed by a board consisting of representatives of renowned academic institutions, R\&D experts, professionals in technology transfer and R\&D investment. ReMedys works, as an expertise hub, based on strong partnerships, to coordinate and drive a virtual $R \& D$ process, closely together with the originators of projects, patients and clinicians. We believe it is essential to keep the accumulated scientific know-how in the projects and to produce what patients and clinicians need via sharing with our partners our drug development know-how and expertise. Our priority goal is to add therapeutic value to our projects, and thus consequently genuine commercial interest. In cases where new therapies, originated at academic institutions, are developed to marketed drugs, it is usually thanks to the pharmaceutical industry stepping in. Any industry interest in any project is proportional to the commercial prospect, leaving smaller indications, such as rare diseases, or innovative but risky therapeutic approaches without support. The pharmaceutical industry is under significant public pressure due to perceived excessive margins, inefficiencies, and for taking advantage of the distress of patients, directly or indirectly. Rare are the innovative models out there to help patients, especially in rare indications, to get access to reasonably priced therapies, and academia to become more successful in advancing towards the clinic innovative ideas. We, in ReMedys, strongly believe that based on smart partnerships, we can build a paradigm shift in the R\&D process, together with all those who are eager to bring therapeutic solutions to patients with high unmet need. Here, we present our effort to reposition a drug for the treatment of amyotrophic lateral sclerosis.

E-Mail: kostas.kaloulis@remedys.net

\section{A019 \\ Muscle Gene Transfer Mediated by Episomal Plasmid Vectors and the PiggyBac Transposon System}

P.S. Iyer, R. van Zwieten, D. Ley, S. Puttini, N. Mermod

Institute of Biotechnology, University of Lausanne, Lausanne, Switzerland

Background: Duchenne muscular dystrophy (DMD) is a frequently occurring lethal X-linked disease in which dysfunctional dystrophin leads to muscle wasting. Autologous transplantation of myogenic stem cells genetically modified to express a functional dystrophin is an attractive therapeutic option for treatment of the disease. There has been very modest success in cell therapy for muscular dystrophy due to several factors; some of them being the difficulty to achieve efficient gene transfer due to the large size of the dystrophin gene and the transgene silencing. The present study investigates the potential use of non-viral gene transfer in mesoangioblasts for cell therapy for DMD. Mesoangioblasts are muscle 
progenitor cells in the embryonic dorsal aorta wall of vertebrates, including mice and humans, with reported ability to fuse with myofibers. Methods: This study focuses on 2 approaches for muscle gene expression with mesoangioblasts. The first approach is using non-replicating episomal plasmid vectors, which are hypothesized to remain in the post-mitotic muscle tissue. The second approach is to induce genomic integration of the transgene by the PiggyBac transposon system. Muscle gene expression is studied by transplantation of genetically modified mesoangioblasts in the tibialis anterior of mice. Results: Intramuscular transplantation of mesoangioblasts containing the integrating transposon system or the episomal plasmid in mouse tibialis anterior muscles led to widespread and sustained eGFP expression in myofibers for at least up to 2 months after transplantation. It is likely that the episomal plasmid follows the same mechanism of long-term persistence as for muscle electroporated in vivo, but without the limitations of in vivo electroporation. In case of the transposon-based approach, transposition in mesoangioblasts allowed sustained expression in mouse muscle, even after mesoangioblast fusion with resident myofibers. Conclusions: These findings provide a proofof-principle that the episomal plasmid vectors as well as the PiggyBac transposon system may have the potential to improve cellbased therapies of DMD. The same approaches will be further used with dystrophin-containing vectors to test improvement of functionality of murine muscles and confirm potential use in therapy. It is hypothesized that the mesoangioblasts expressing dystrophin would be able to fuse with the myofibers and improve muscle functionality.

E-Mail: pavithra.iyer@unil.ch

\section{A020 \\ Derivation of Traceable and Transplantable Photoreceptors from Mouse Embryonic Stem Cells}

S. Decembrini, U. Koch, F. Radtke, A. Moulin, Y. Arsenijevic

Unit of Gene Therapy \& Stem Cell Biology, University of Lausanne, Lausanne, Switzerland

Retinal degenerative diseases resulting in the loss of photoreceptors are a major cause of blindness. Recently, different groups validated the possibility to reactivate dormant retinal circuits of degenerating retinas using retinal prosthesis, gene therapy, and cell replacement therapy. Photoreceptor replacement therapy may be feasible since transplanted photoreceptors, collected directly from the developing or the adult retina, have been shown to restore some visual function in mice affected by retinal degeneration. Because the developing retina is not a suitable source of renewable photoreceptors, we focused on embryonic stem cells (ESC) for their capacity to generate retinal progenitors and photoreceptor cells in vitro. In this study, we derived a new transgenic ESC line in which the reporter gene, the Crx-GFP transgene, is expressed in both post-mitotic immature and mature photoreceptors, and assessed the extent to which this protocol recapitulates photoreceptor development in vitro. Various oxygen concentrations were tested at different development stages to improve photoreceptor production. As observed during retinogenesis, the optimized 3Dretina induction protocol allows the production of GFP-positive photoreceptors between 12 and 14 days of culture reaching the peak of birth between day 18 and 20 of culture. Similarly the intensity of the GFP signal and their alignment increased over time. We observed that hyperoxic condition improved photoreceptor survival only when present from photoreceptor differentiation onset. Up to 10 layers of photoreceptors can be formed in each in vitrogenerated retina. In addition, we proved that transplantation of ESC-derived photoreceptors is feasible. No appearance of tumor formation was detected after transplantation of sorted photoreceptor cells. Many Crx-GFP-positive cells show the presence of outer-segments, ribbon synapses and light signal transduction pathway proteins. These experiments show the feasibility to reliably generate a large quantity of integration-competent photoreceptors from ESC. A further characterization of the transplanted photoreceptors to reveal their capacity to mediate light stimuli is underway.

E-Mail: sarah.decembrini@fa2.ch

\section{A021 \\ Fanconi Anemia: From Gene Discovery to Gene Therapy}

J. Surralles

Department of Genetics and Microbiology, Universitat Autònoma de Barcelona, and Center for Biomedical Network Research on Rare Diseases, Barcelona, Spain

Background: Fanconi anemia (FA) is a rare genetic disease characterized by progressive anemia, malformations, chromosome fragility, hypersensitivity to DNA interstrand cross linkers (ICL) and cancer predisposition. The only cure of the hematological disease is bone marrow transplantation using an HLAcompatible donor. Those families with unavailable donor rely on preimplantation genetic diagnosis with selection of an HLA-related embryo and in the future implementation of advanced gene and cell therapies. In this context, we previously generated disease-free hematopoietic progenitors from the skin of FA patients, and we are running a phase I/II gene therapy clinical trial using a lentiviral vector carrying the FA gene FANCA designated as orphan drug by the European Medicines Agency. All these novel therapeutic applications to human health are further complicated by the fact that at least 16 FA genes have been identified, some of them involved in breast cancer susceptibility in otherwise unaffected mutation carriers. Therefore, the discovery of novel FA genes is important not only for the affected patients but also for the general population and for the future implementation of advanced gene/cell therapies. Methods: $110 \mathrm{FA}$ patients were genetically studied by retroviral subtyping and mutational screening, and those unassigned patients were subjected to whole-exome sequencing. The candidate gene was functionally studied at the gene and protein level. Results: A novel FA gene (ERCC4/FANCQ) encoding the DNA repair nuclease XPF was discovered. This gene causes not only FA but also Xeroderma pigmentosum, Cockayne syndrome and XFE-type progeroid syndrome, 3 clinical conditions characterized by defects in nucleotide excision repair (NER) of UV-light-induced DNA damage. We demonstrated that mutations leading to FA specifically disrupt ICL but not NER due to abnormal nuclease activity of the mutant protein. Conclusion: ERCC4/FANCQ mutations cause 4 rare diseases depending on the 
resulting balance between ICL repair and NER deficiencies, highlighting the complex interplay between genotype and phenotype in human health and disease.

E-Mail: jordi.surralles@uab.es

\section{A022 \\ Mutation Analysis in Two Swiss Families with Non-Syndromic Hereditary Hearing Impairment through Sequence Capture and Next-Generation Sequencing}

N. Gürtler ${ }^{\mathrm{a}}$, K. Ludin ${ }^{\mathrm{b}}$, B. Röthlisberger ${ }^{\mathrm{b}}$

aDepartment of Otolaryngology, University Hospital of Basel, Basel, and bDepartment of Genetics, Cantonal Hospital Aarau, Aarau, Switzerland

Background: Hereditary non-syndromic hearing impairment is characterized by a high heterogeneity with dozens of genes and hundreds of different mutations. The search for mutations by classic sequencing methods is time- and labor-intensive. New methods such as next-generation sequencing based on higher throughput and array technology offer a much higher yield. The objective was to detect mutations by targeted genome capture in combination with next-generation sequencing in 2 Swiss families with hereditary non-syndromic hearing impairment. Hearing impairment in family A is sensorineural, postlingual and affects the high frequencies. Family B shows sensorineural, postlingual hearing impairment in the low frequency range. Methods: First, a possible larger pathogenic deletion was investigated with an exon-focused high-density Roche NimbleGen CGH array. Second, a custom Sequence Capture 385K Human Array was designed and manufactured by Roche NimbleGen. The array included all known genes associated with hereditary hearing loss. After capturing the targeted regions, the DNA was eluted, amplified and then sequenced on an Illumina MiSeq instrument. Variants were detected with the SEQNext Software (JSI Medical Systems) and possible pathogenic mutations were verified by Sanger sequencing in the patient and further family members. Results: The mutation c. $5383+5$ delGTGA in the TECTA gene was discovered in family A. The mutation was present in 8 affected and absent in 7 non-affected family members, consistent with segregation of hearing impairment. Family B harbored a novel mutation in the WFS1 gene: c.2614-2625delCATGGCGCCGTG (p.872-875delHGAV), which segregated with affected family members. Conclusions: The search for mutations in hereditary hearing impairment can be facilitated by novel methods such as targeted genome capture in combination with next-generation sequencing.

E-Mail: Nicolas.guertler@usb.ch

\section{A043 \\ Immune-Mediated Activation of Coagulation in Stevens Johnson Syndrome/Toxic Epidermal Necrolysis \\ O. Iqbal ${ }^{\mathrm{a}, \mathrm{b}}$, D. Syed ${ }^{\mathrm{a}}$, J. Malalis ${ }^{\mathrm{b}}$, C. Mata ${ }^{\mathrm{b}}$, A. Lin $^{\mathrm{b}}$, M. Mosier , E. Campbell' , S. Abro a , D. Hoppensteadt ${ }^{\mathrm{a}}$, J. Fareed ${ }^{\mathrm{a}}$, C. Bouchard ${ }^{\mathrm{b}}$}

Departments of apathology, ${ }^{b}$ Ophthalmology, 'Surgery, and

${ }^{\mathrm{d}}$ Molecular \& Cell Physiology, Loyola University Health System, Maywood, III., USA

Background: Stevens Johnson syndrome/toxic epidermal necrolysis (SJS/TEN) are specific drug hypersensitivity reactions initiated by cytotoxic T-lymphocytes. Augmentation of T-cell activation by positive co-stimulation and major histocompatibility complex-restricted presentation of the culprit drug triggers T-cell activation following interaction with $\mathrm{T}$-cell receptors. This $\mathrm{T}$-cell activation results in expression of cytokines including TNF- $\alpha$, interferon- $\gamma$ and granzyme B from NK cells. At the immunity-coagulation interface, these cytokines cause coagulation activation. Materials and Methods: Following an Institutional Review Board approved protocol, blood samples were obtained from 11 subjects suspected of SJS/TEN and 2 normal healthy volunteers. Confirmatory biopsies were performed in all SJS/TEN subjects that confirmed SJS/TEN in 4 subjects and were negative in 7 subjects. Immunohistochemistry was performed on the skin biopsy sections using anti-granulysin, FasL and anti-granzyme B antibodies. Cytokine levels were measured using the cerebral array II biochip (Randox Laboratories Limited, Crumlin, UK). Besides cerebral array II, other parameters such as thrombin-antithrombin (TAT) complexes (Dade ${ }^{\circledR}$, Marburg, Germany), fibrinopeptide A (F1.2; Dade), plasminogen activator inhibitor-1 (PAI-1; Stago ${ }^{\circledR}$ ) and STACHROME antithrombin (Stago), ZYMUPHEN platelet microparticles activity (Hyphen ${ }^{\circledR}$ Biomed, Neuville-sur-Oise, France), and HEMOCLOT protein C (Stago) were measured using ELISA kits as per manufacturers' instructions. Results: Compared to the normal human plasma, the IL-4, IL-6, TNF- $\alpha$, and MCP-1 levels were increased significantly in biopsy-confirmed SJS/TEN patients $(\mathrm{p}=0.0094,0.0276,0.0138$, and 0.0025 , respectively). Other parameters measured including IL-2, IL-8, IL-10, VEGF, INF- $\gamma$, IL- $1 \alpha$, IL1- $\beta$, and EGF were not significantly increased. A marked increase in the TAT complexes $(6.3 \pm 5.9 \mu \mathrm{g} / \mathrm{ml}), \mathrm{F} 1.2(430.4 \pm 202.4 \mathrm{pmol} / \mathrm{l})$, platelet microparticles $(13.1 \pm 9.3 \mathrm{nM})$, and protein C levels $(90.5 \pm$ $63.4 \%$ ) with a corresponding decrease in PAI-1 $(53.3 \pm 18.8 \mathrm{ng} / \mathrm{ml})$ and antithrombin levels $(80.7 \pm 42.4 \%)$ compared to normal human plasma were also observed. Biopsy-negative SJS/TEN subjects with less pronounced inflammatory stimulus demonstrated mild elevation in cytokine levels of IL-4 (1.95 $\pm 0.59 \mathrm{pg} / \mathrm{ml}), \mathrm{IL}-6$ (29.81 \pm $25.18 \mathrm{pg} / \mathrm{ml})$, TNF- $\alpha(7.20 \pm 5.04 \mathrm{pg} / \mathrm{ml})$, and MCP-1 $(265.10 \pm$ $159.09 \mathrm{pg} / \mathrm{ml}$ ). Immunohistochemical studies revealed high expression of granulysin and granzyme B. Conclusions: T-cell activation and release of cytokines, especially TNF- $\alpha$, granulysin and granzyme B evidenced by their increased expression in immunohistochemistry, causes immune-mediated activation of coagulation with increase in TAT, MCP-1, F1.2, and platelet microparticles and corresponding decrease of protein $\mathrm{C}$, antithrombin and PAI-1. These alterations in coagulation may progress to sepsis-associated coagulopathy and overt disseminated intravascular coagulation.

E-Mail: OIQBAL@lumc.edu 